\title{
Les collections « spécialisées » à la Bibliothèque nationale de France : un bref panorama
}

\section{Denis Bruckmann}

\section{OpenEdition}

1 Journals

Édition électronique

URL : http://journals.openedition.org/rbnu/595

DOI : $10.4000 /$ rbnu.595

ISSN : 2679-6104

Éditeur

Bibliothèque nationale et universitaire de Strasbourg

\section{Édition imprimée}

Date de publication : 1 novembre 2018

Pagination : 8-19

ISBN : 9782859230784

ISSN : 2109-2761

\section{Référence électronique}

Denis Bruckmann, «Les collections «spécialisées » à la Bibliothèque nationale de France : un bref panorama », La Revue de la BNU [En ligne], 18 | 2018, mis en ligne le 01 juillet 2019, consulté le 10 décembre 2020. URL : http://journals.openedition.org/rbnu/595; DOI : https://doi.org/10.4000/rbnu. 595

\section{(c) (ஒ)(}

La Revue de la BNU est mise à disposition selon les termes de la Licence Creative Commons Attribution - Pas d'Utilisation Commerciale - Partage dans les Mêmes Conditions 4.0 International. 

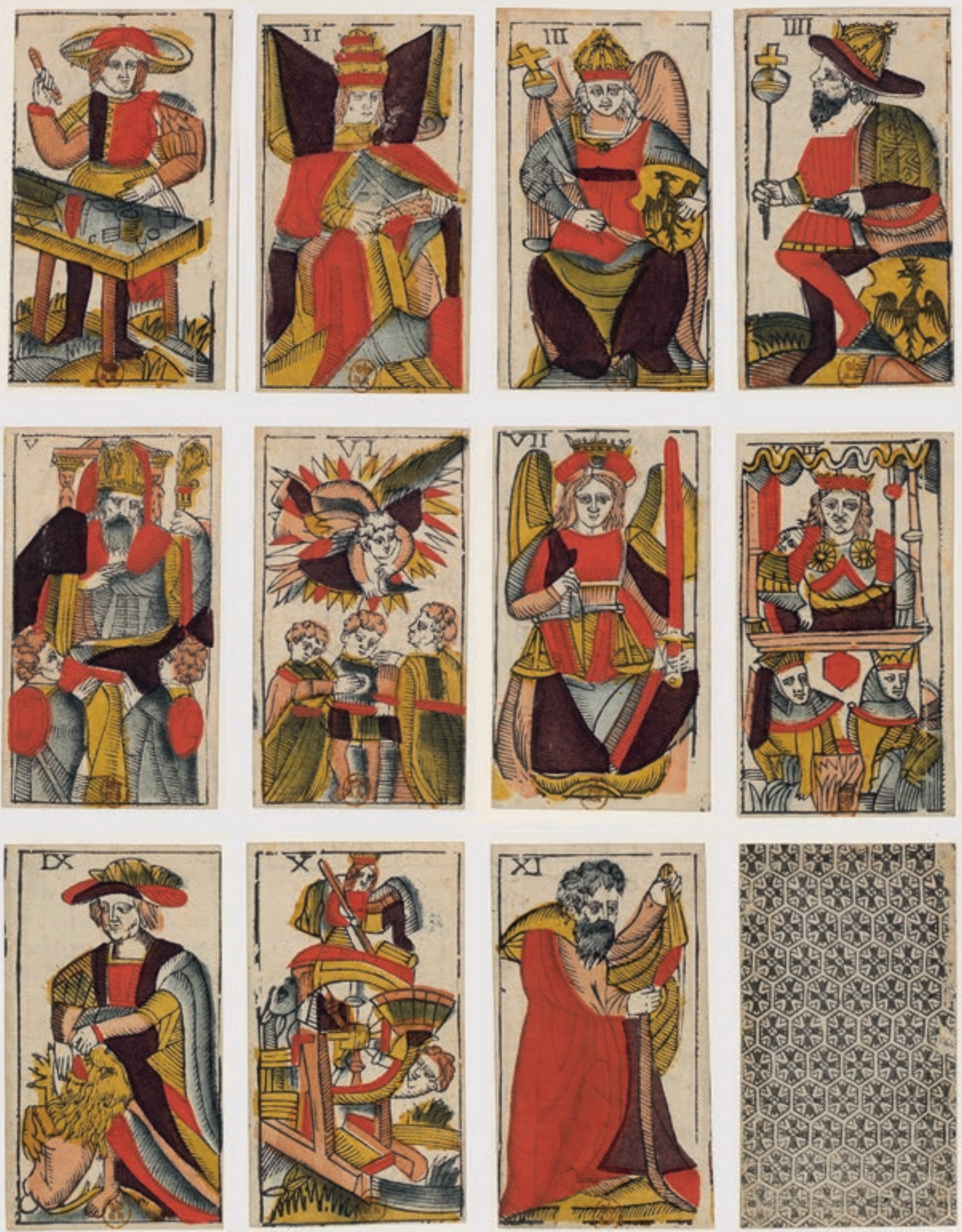

Tarot Viéville (Paris) : les onze premiers atouts d'un jeu de cartes du $17^{e}$ siècle, dont le département des Estampes détient une remarquable collection de toutes les époques. Un beau livre est à paraitre sur ces collections (coll. BnF). 


\section{LES COLLECTIONS «SPÉCIALISÉES " À LA BIBLIOTHĖQUE NATIONALE DE FRANCE : UN BREF PANORAMA} PAR DENIS BRUCKMANN

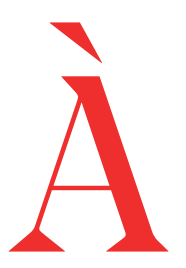

à la bibliothèque

la Bibliothèque nationale $(\mathrm{BN})$, dont on fait remonter la création à $1368^{2}$, puis à la Bibliothèque nationale de France (BnF), créée en 1994, la présence de documents autres que les livres dans les collections ne relève pas de hasards ou d'opportunités, mais bien de l'identité de la bibliothèque, d'une façon qu'on pourrait dire consubstantielle. C'est une histoire très longue et très complexe. Elle enchevêtre bibliothèques personnelles des souverains et bibliothèque accessible aux savants ou au public, dépôt légal et entrées onéreuses ou gracieuses selon tous les scénarios juridiques possibles ${ }^{3}$, entrées d'œuvres ou de documents mais aussi entrées de collections entières, patrimoine national ou régional et entrées étrangères d'horizons proches ou très lointains. On pourrait y ajouter une chronique administrative très riche qui ne va pas sans rapprochement ou fusion d'institutions, fréquentes réformes d'organisation, création ou division de multiples départements.
L'intention de cet article n'est pas de retracer, même brièvement, cette histoire qui d'ailleurs comporte de très nombreuses zones d'ombre, mais de donner une idée générale de l'importance de ces collections pour l'établissement et les publics.

Commençons par le début (qui est aussi le plus complexe) et les entrées hors du cadre du dépôt légal. Selon Simone Balayé, une des grandes spécialistes de l'histoire de la $\mathrm{BN}$, aux origines, la bibliothèque se distingue mal du cabinet de curiosités, et longtemps il n'est pas facile non plus de distinguer bibliothèque personnelle du souverain et bibliothèque comme institution. Dès le Moyen-Âge, la plupart des rois de France (Philippe Auguste, Jean Le Bon, Charles V) disposent d'une bibliothèque où sont rassemblés livres (à l'époque manuscrits), mais aussi orfèvrerie, pierres gravées, monnaies, bijoux, curiosités naturelles des règnes animal ou minéral. Ces collections sont transmises à la mort des souverains, mais souvent partagées entre les héritiers. L'idée d'une continuité ne se forme que peu à peu, avec Louis XI, puis François $1^{\text {er }}$ (qui installe sa bibliothèque à Fontainebleau et, le premier, lance 
des missions de collecte à l'étranger, notamment de manuscrits grecs), Charles IX (qui créera le Cabinet des médailles, d'abord sans rapport avec la bibliothèque royale), et Henri IV enfin ; tels sont les quelques rois qu'on évoque plus particulièrement quand on essaie de décrire le développement de la bibliothèque comme institution.

C'est sous Louis XIV que se produit le grand tournant, grâce à l'impulsion de Colbert. La collection d'objets, de monnaies et de médailles du roi s'enrichit en 1660 de la collection personnelle de Gaston d'Orléans, frère de Louis XIII. Cette dernière consolide également la présence des cartes à la bibliothèque royale (amorcée un peu plus tôt), grâce à 1800 planches de cartes géographiques, portraits de ville, plans de fortifications et de sièges. En 1666, la bibliothèque s'installe rue Vivienne (très près de ce qu'on considère être son berceau historique, rue de Richelieu). En 1667, c'est l'acquisition par la bibliothèque royale de la très importante collection d'estampes de Michel de Marolles - plus de 120000 pièces - qui servira de noyau à la création du Cabinet des estampes. 1667 voit aussi l'entrée de la collection de manuscrits arabes, persans, turcs, hébreux et grecs de Gilbert Gaulmin, maître des requêtes, qu'on considère comme le premier acte d'une politique cohérente d'accroissement des collections orientales de la bibliothèque. En 1720 sont créés les premiers grands départements : Imprimés (les livres), Manuscrits, Médailles et pierres gravées, Planches gravées et recueils d'estampes, Titres et généalogies, département qui sera ultérieurement rapproché des Manuscrits. Tout au long du $18^{\mathrm{e}}$ siècle, la diversification des collections se poursuit et s'accroît encore. En 1726 entrent les collections musicales de Sébastien de Brossard, et l'année suivante les manuscrits autographes de Marc-Antoine Charpentier. Si les livres de musique n'étaient pas absents de la bibliothèque royale, on considère que ces entrées sont le premier témoignage d'un intérêt marqué pour la musique en son sein. Par ailleurs, l'archéologie est en train de naître. Les spécialistes de l'Antiquité trouvent à la bibliothèque un foyer de recherches et de la documentation, qu'ils augmentent de nombreux dons, parfois de plusieurs milliers d'objets comme c'est le cas pour un des plus célèbres d'entre eux, le comte de Caylus. Du côté des manuscrits, les collections se complètent par l'acquisition de la remarquable collection personnelle de Colbert (en 1732, plus de 6000 volumes) et s'étendent toujours plus loin à l'Est : livres chinois, vietnamiens, indiens... On a aussi des preuves que dès avant la Révolution, étaient présents dans les collections des instruments scientifiques liés à la géographie et à l'astronomie, sans compter les globes attestés assez tôt dans les collections royales.

La période moderne a ajouté encore à la présence des collections dites « spécialisées ». On ne peut pas ne pas mentionner, en 1881, le legs de ses manuscrits par Victor Hugo à la Bibliothèque nationale, qu'il imagine devenir « un jour la bibliothèque des États Unis d'Europe ", et qui fondera la tradition de la collecte des manuscrits d'écrivains pour la plupart entrés dans les collections rétrospectivement. Le $19^{\mathrm{e}}$ siècle verra aussi l'extension de la collecte des manuscrits aux papyrus égyptiens, aux codices mexicains, aux manuscrits éthiopiens... avant l'entrée en 1910 de 10000 manuscrits chinois, tibétains, sanscrits et autres encore, grâce à la mission de Paul Pelliot.

En 1920, le banquier Auguste Rondel donne à l'État son extraordinaire collection sur les arts du spectacle (théâtre, cinéma, cirque, mime, etc.). Au-delà des manuscrits, livres et revues, il a collecté infatigablement iconographie, archives, petite documentation - programmes, affiches, invitations. Cette collection sera le socle du futur département des Arts du spectacle (créé en 1976). Les arts du spectacle constitueraient d'ailleurs un excellent sujet d'étude sur le développement d'une politique patrimoniale autour d'un objet complexe. Partie de matériaux documentaires imprimés assez traditionnels, l'ambition de collecte n'a cessé de s'étendre à travers dons, acquisitions, legs, et a concerné archives de toutes sortes (dès la collection Rondel), puis matériaux audiovisuels, photographies et maquettes de costumes ou de décors dans les années cinquante, marionnettes, masques, costumes par la suite, notamment grâce à l'acquisition des archives du célèbre metteur en scène Edward Gordon Craig (en 1957), ou encore, plus tard, éléments plus amples sur les équipements des salles de spectacle (collection Clémançon).

En 1934, la bibliothèque de l'Arsenal est rattachée à la Bibliothèque nationale. Cette bibliothèque dont la principale origine est la collection du marquis de Paulmy, passée ensuite au comte d'Artois, ajoute à la Bibliothèque nationale de magnifiques ensembles de 


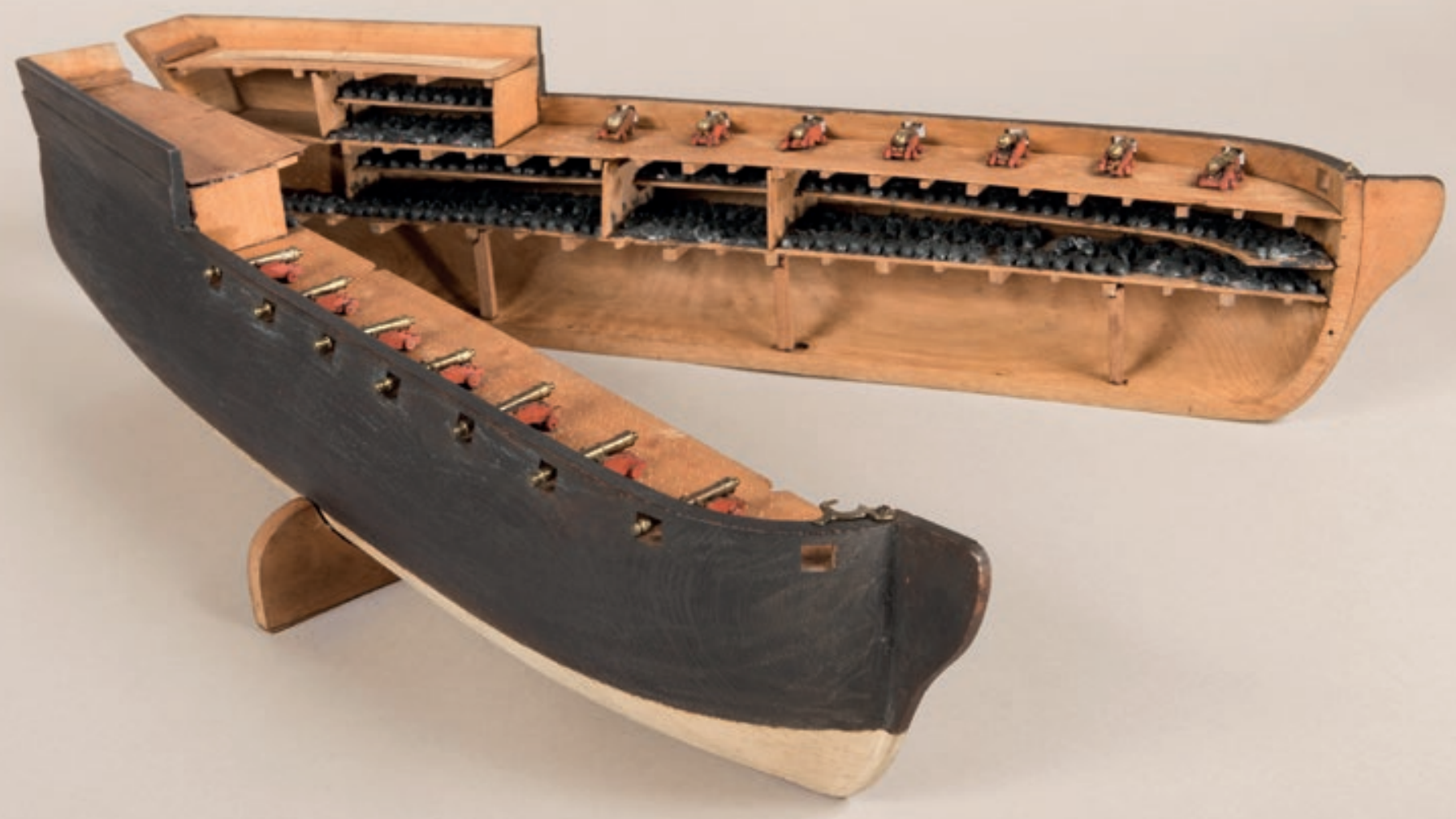

Maquette de bateau négrier, commandée par Mirabeau pour plaider l'abolition de l'esclavage à l'Assemblée (1789; coll. Bibliothèque de l'Arsenal)

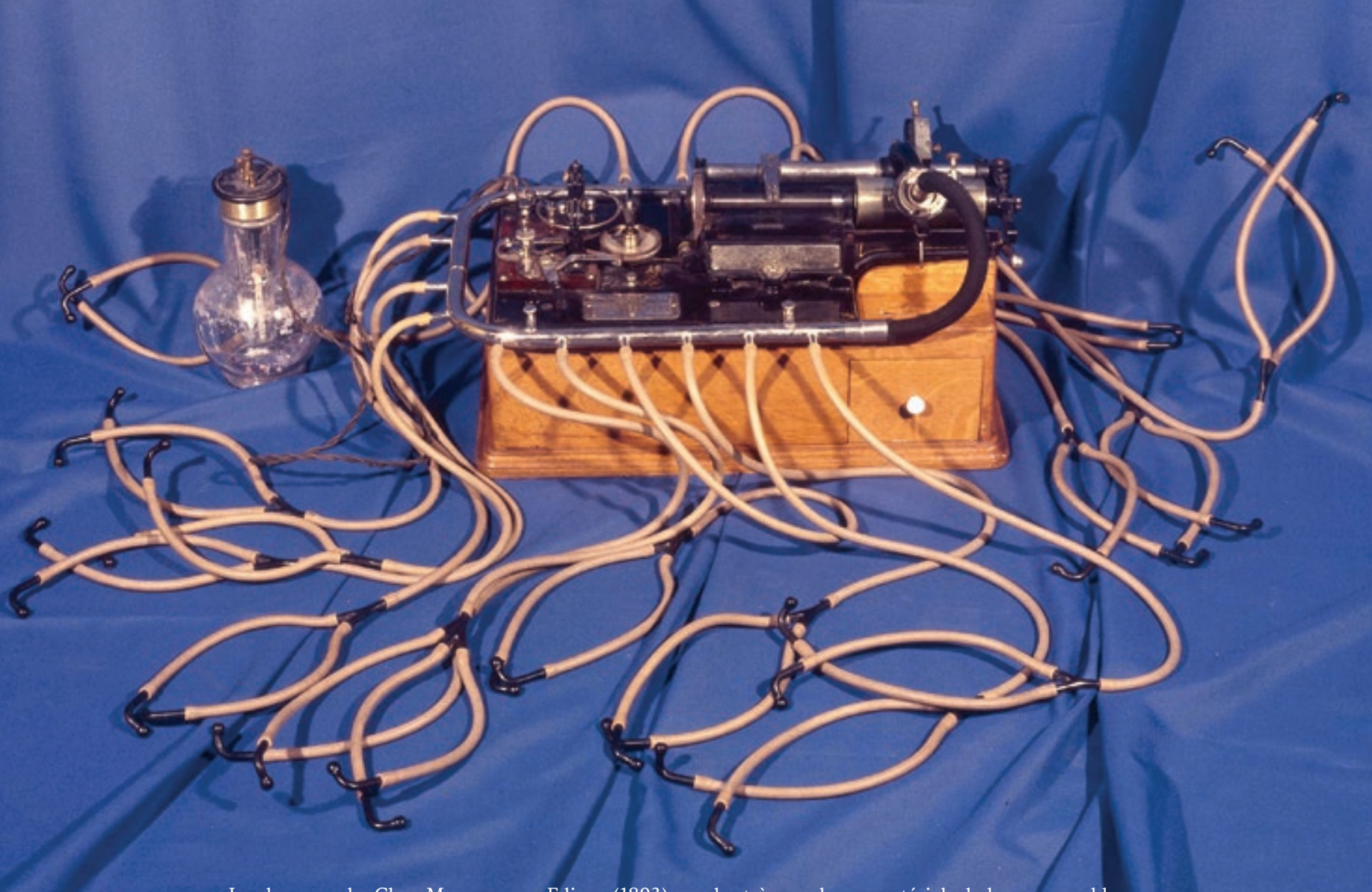

Le phonographe Class $\mathrm{M}$, conçu par Edison (1893), un des très nombreux matériels de la remarquable collection Charles Cros (département de l'Audiovisuel) 


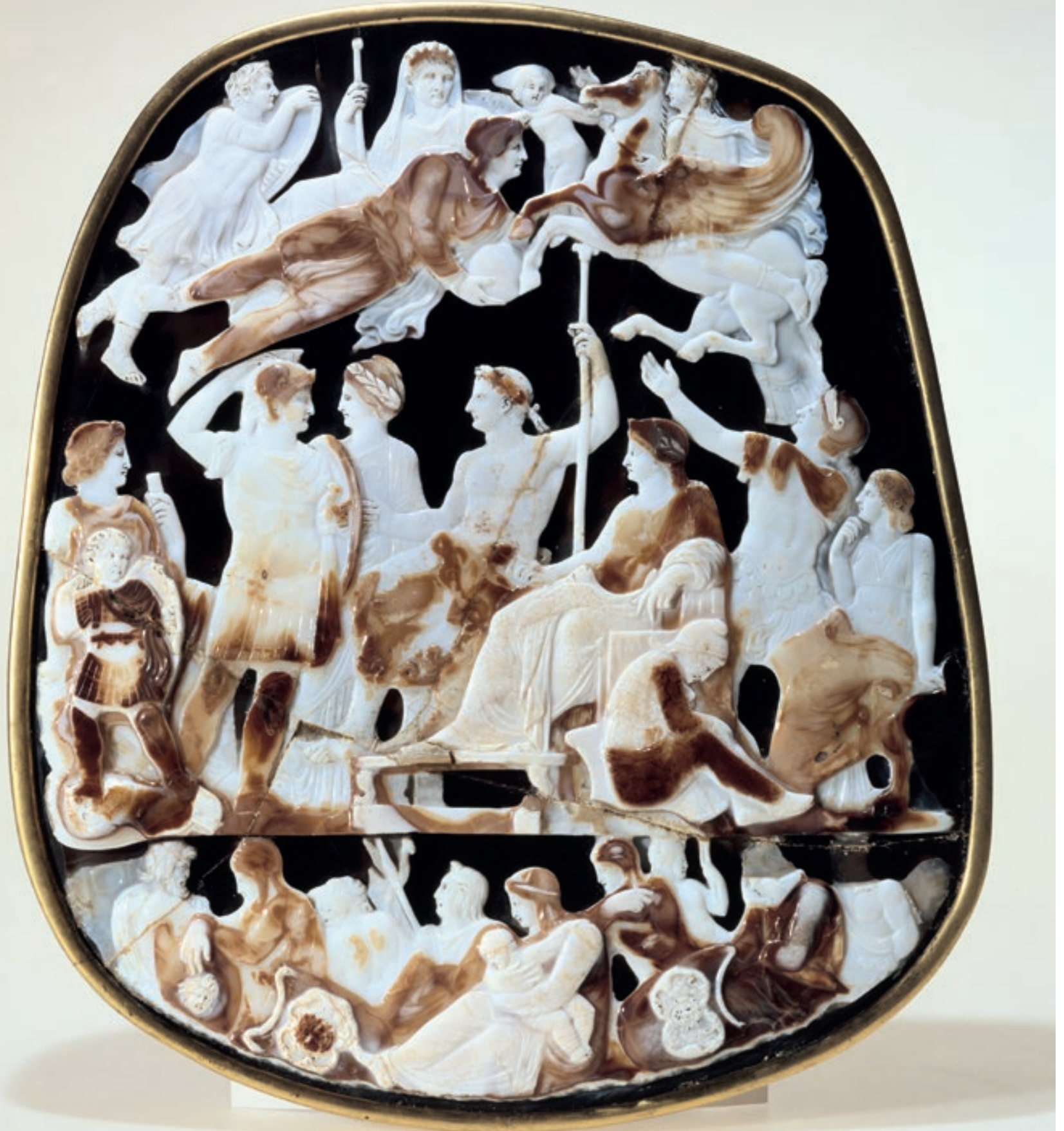

Le grand Camée de France (la glorification de Germanicus), acheté par saint Louis à Constantinople, en 1247. Plus grand camée antique du monde $(30 \mathrm{~cm})$, il est entré dans les collections de la Bibliothèque nationale en 1791, sur ordre de Louis XVI (département des Monnaies, médailles et antiques). 
gravures, de cartes et plans, de manuscrits médiévaux et modernes, ainsi qu'un intéressant fonds musical. En 1935, c'est au tour de la Bibliothèque-musée de l'Opéra, fondée en 1866, de se voir rattachée à la $\mathrm{BN}$ : ce sont presque trois siècles d'archives et de documents divers liés aux spectacles de l'Opéra qui entrent dans les collections : maquettes de décors, très nombreux tableaux, objets divers. Il en va de même, à la même date, pour la bibliothèque du Conservatoire, très riche en manuscrits musicaux prestigieux et dans une moindre mesure en iconographie. Avec l'entrée de ces deux collections, la musique devient un point fort de la $\mathrm{BN}$ qui crée, quelques années plus tard, son premier département thématique, le département de la Musique. En 1942, c'est la Société de géographie qui procède au dépôt de ses extraordinaires collections de manuscrits, de cartes, de photographies, de globes, lesquelles sont intégrées au département des Cartes et plans.

Mais la grande affaire de la période moderne est l'entrée de l'audiovisuel. En 1911, le linguiste Ferdinand Brunot fonde les "Archives de la parole ", avec l'aide de l'industriel Émile Pathé, afin d'enregistrer voix célèbres et parlers locaux. Ce laboratoire de recherche se mue en " Musée de la parole et du geste " en 1927. À sa création en 1938, la «Phonothèque nationale " est d'abord installée au Musée de la Parole, qu'elle finira par absorber. Cette phonothèque rejoint la Bibliothèque nationale en 1975, et au moment même se crée le dépôt légal des vidéogrammes et des documents multimédia. $\mathrm{Au}$-delà des documents audiovisuels, ces institutions successives conservent une bonne partie des matériels de lecture nécessaires pour les consulter. L'ensemble - du phonographe à la console de jeu vidéo, en passant par ordinateurs, caméras, microphones - constitue l'une des collections les plus intéressantes et les moins connues de la $\mathrm{BnF}$, la collection Charles Cros, riche d'environ 1500 matériels.

Du côté du dépôt légal, les choses sont apparemment plus simples. Créé en 1537 par François $1^{\text {er }}$ dans la célèbre ordonnance de Montpellier, le dépôt légal répond à une double visée, de surveillance des idées certes, mais aussi patrimoniale ${ }^{4}$. L'histoire du dépôt légal est celle d'une extension à toutes les productions éditoriales, en fonction des évolutions technologiques qui ont affecté celles-ci. Tombent successivement sous l'obligation de dépôt légal, les estampes et les cartes et plans (en 1648), les partitions musicales (en 1793), la monnaie et les médailles (selon plusieurs textes de la période révolutionnaire et du début de l'Empire), les photographies et les phonogrammes (en 1925), les affiches (en 1941), les vidéogrammes et les multimédias composites (en 1975), les logiciels, bases de données et documents électroniques (en 1992). Le dernier dépôt légal créé en France l'est en 2006 : celui de l'Internet « français $»^{5}$.

L'« efficacité » du dépôt légal est selon les types de documents en retard ou en avance sur sa création. Il a fallu souvent plusieurs décennies pour que le dispositif légal entre dans les faits. Il en est ainsi pour le dépôt légal des imprimés qui n'a vraiment fonctionné qu'à partir du $17^{\mathrm{e}}$ siècle, et surtout pour les publications savantes, pour les cartes - dépôt légal renforcé en 1672 - ou encore pour les disques, le premier phonogramme étant entré par dépôt légal à la Phonothèque nationale en 1940, suite à un nouveau décret en 1938. Pour la photographie, en revanche, il se met en place spontanément dès 1851, très en amont du texte de loi. Dans la rivalité avec la gravure pour documenter le réel, les photographes ont très tôt déposé leur production à la Bibliothèque nationale pour faire reconnaître la nouvelle technique, ce qui a permis la création d'une des plus anciennes et des plus diverses collections de photographies au monde. Il en va de même pour les affiches.

Toutes ces sources (et leurs affluents) ont engendré un immense fleuve, une «bibliothèque monde " : environ 30 à 40 millions de documents (si l'on y inclut les documents numériques) conservés sur plusieurs sites : 15 millions d'images fixes, 2 millions de partitions, 1500000 enregistrements sonores, 900000 cartes, plans et globes, 440000 monnaies et jetons, près de 300000 vidéogrammes, 170000 multimédias ${ }^{6}, 150000$ médailles, plus de 60000 objets divers dont plus de 40000 objets archéologiques, plusieurs milliers de maquettes en trois dimensions, 6500 costumes, 1500 marionnettes, plus de 500 masques, sans parler des objets encore insuffisamment dénombrés dont les puzzles, les modèles réduits, les maquettes à monter, tous les petits matériaux d'accompagnement de certaines publications (petits jouets, figurines, 
gadgets) - en définitive, les «non livres ", comme on appelait ce type de matériaux dans les années soixante, représentent une majorité assez large des collections. Et s'il faut le redire, une très grande partie de ceux-ci sont arrivés par le biais de dons de créateurs, d'ayants droit, de collectionneurs.

S'il fallait choisir quelques exemples sans composer une liste à la Prévert, que choisir ? La première monnaie frappée sur le sol de France, vers 500 avant Jésus-Christ, le grand Camée de France, un chefd'œuvre absolu de l'Antiquité, acheté par saint Louis à Constantinople en 1247, un coffret dit de pèlerinage $\mathrm{du} 15^{\mathrm{e}}$ siècle contenant une maladroite mais émouvante gravure sur bois, le globe vert, du début du $16^{\mathrm{e}}$ siècle, soit le premier globe où figure la représentation de l'Amérique, un des très beaux jeux de cartes du $17^{\mathrm{e}}$ siècle que conserve le département des Estampes, le manuscrit du Don Giovanni de Mozart, l'émouvante maquette de bateau négrier réalisée à la demande de Mirabeau pour plaider l'abolition de l'esclavage à l'Assemblée, la collection de bijoux de scène de l'Opéra, un des premiers phonographes d'Edison, un agenda de Proust, un petit carnet de Georges Bataille ou encore la petite robe noire d'Edith Piaf (objets pour la plupart reproduits ici) ? Il faudra un jour publier un voyage dans les collections spécialisées de la BnF et dire tout ce qu'elles apportent à la culture et à la recherche.

Dans un article à vocation de panorama, il paraît difficile d'aller plus loin. Au-delà des quelques pistes bibliographiques données en annexe, il est conseillé de s'adresser aux différents départements pour obtenir des informations complémentaires sur l'histoire de telles ou telles collections.

\section{Cohésion ou dispersion?}

L'ampleur de ces collections peut donner le vertige et faire s'interroger sur l'ambition patrimoniale de la bibliothèque et plus largement des politiques publiques, si l'on peut utiliser cette expression pour les siècles passés.

Les questions sur les périmètres de collecte n'ont pas manqué, et notamment dans la période récente - ainsi par exemple celle de la création de la BnF dans les années 1990, où pendant quelques années une réflexion a été menée sur la création d'une Biblio- thèque nationale des Arts, qui en complément de la $\mathrm{BnF}$ (domaine des imprimés) aurait rassemblé l'ensemble des collections spécialisées. Certains départements ont fait l'objet d'une réflexion particulière, pour savoir sur quel site il convenait de les implanter : ainsi le département des Manuscrits, la Réserve des livres rares ou encore le département de l'Audiovisuel, qui ont un peu navigué entre le site Richelieu et le site François Mitterrand. Plus récemment, en 2009, une inspection de l'Inspection générale des finances s'est inscrite dans les annales de la bibliothèque, pour avoir interrogé l'établissement sur le bien-fondé en son sein de la Bibliothèque-musée de l'Opéra, de la Bibliothèque de l'Arsenal, sur l'opportunité du dépôt au Louvre d'une grande partie des objets archéologiques, ou encore, pour prendre un objet plus contemporain, la soutenabilité économique et technique des collectes de l'Internet.

En définitive, ces interrogations récurrentes et légitimes - paraissent plutôt des épiphénomènes. Il est sans doute consubstantiel à la mémoire de vouloir s'enrichir toujours, d'avoir pour ambition l'exhaustivité. Si l'on reprend l'exemple des arts du spectacle, n'est-il pas légitime aussi qu'afin de cerner un domaine aussi riche, tous les matériaux documentaires soient peu à peu pris en compte, au risque d'une inflation difficile à contenir, mais garante d'une plus grande « scientificité » ?

Par ailleurs, comme nous l'avons montré plus haut, ce sont souvent les pouvoirs publics eux-mêmes qui, au fil de l'histoire, ont rattaché à la BN des établissements divers et pleinement indépendants, voyant là une garantie de pérennité, d'expertise, et sans doute des formes de mutualisation de moyens avant l'heure. Globalement, la politique mémorielle du patrimoine imprimé, graphique et audiovisuel français nous est plutôt enviée. Le dépôt légal de François $1^{\text {er }}$ - résurgence géniale de la bibliothèque d'Alexandrie ? - a servi de modèle à beaucoup de dispositifs étrangers, le dépôt légal du Royaume-Uni par exemple, en 1610, celui de la Suède, créé en 1661, ou encore celui du Danemark créé en 1697. Bon nombre de pays "nouveaux " - Canada, États-Unis - s'en sont inspirés pour créer des dispositifs identiques ou voisins - ainsi le " copyright act " américain (en 1790). Et certains collègues d'autres pays comme le Japon par exemple - nous disent combien ils regrettent que leur législation n'ait pas pris en compte les estampes, par exemple, ou encore l'imagerie. 


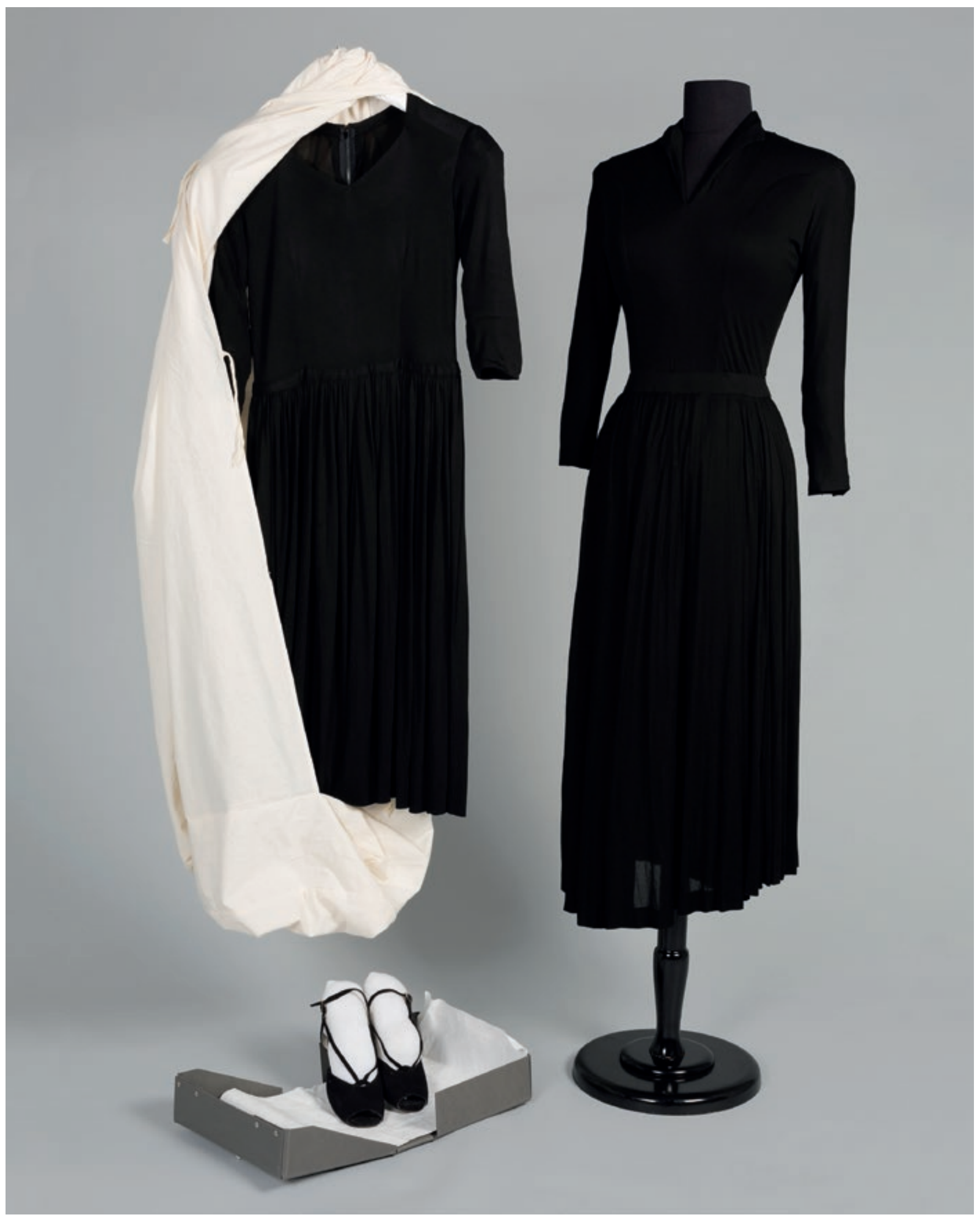

Une des célèbres petites robes noires d'Edith Piaf, donnée à la BnF en 2008 par Danielle Bonnel, la secrétaire et éclairagiste de la chanteuse, avec d'autres objets personnels

(département des Arts du spectacle) 


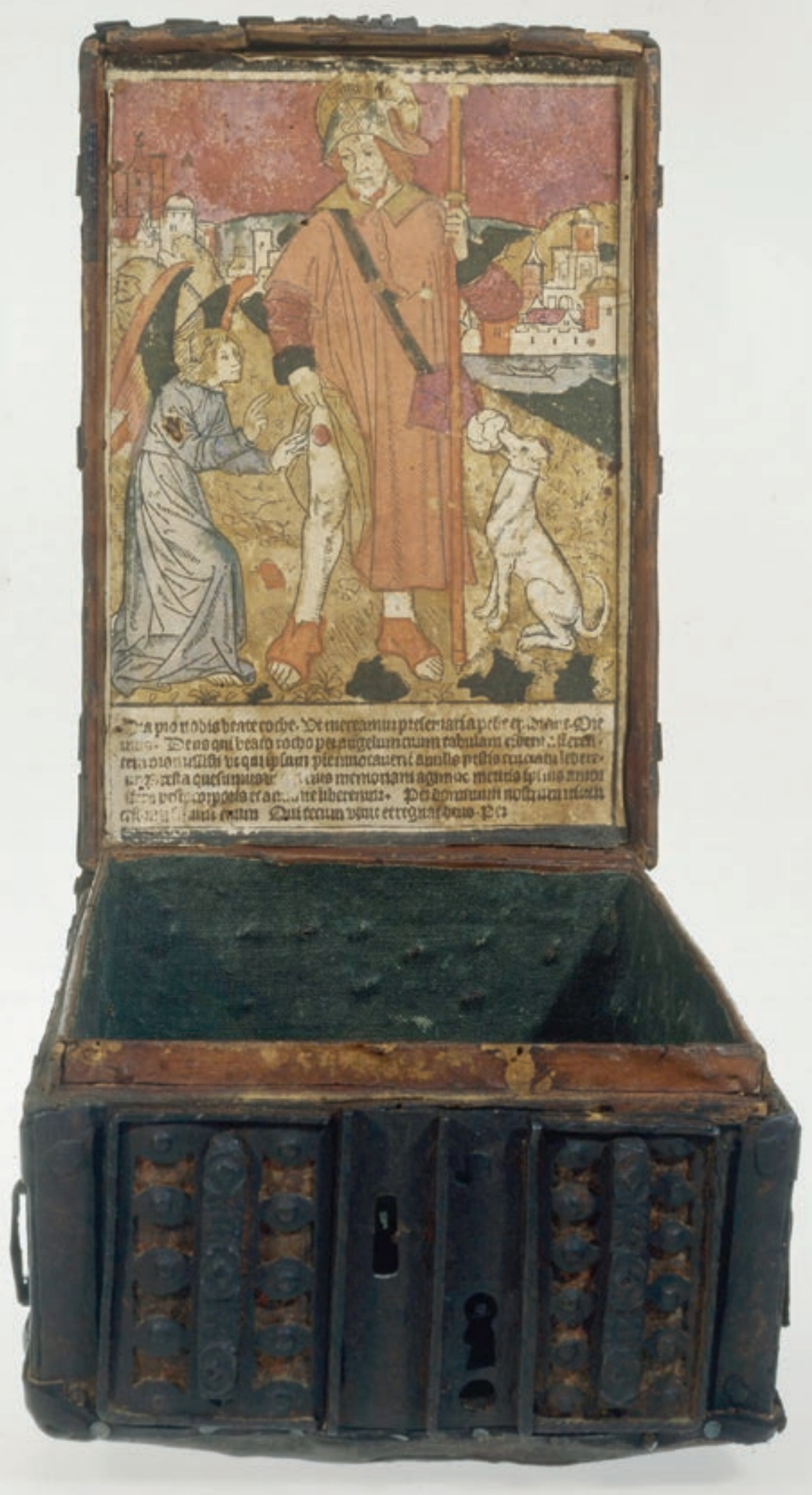

Coffret de pèlerinage orné d'une gravure sur bois : saint Roch découvrant sa plaie (Moyen-Âge). La destination de ces coffrets à des pèlerinages reste aujourd'hui incertaine (département des Estampes et de la photographie). 
La BnF n'en est pas moins confrontée à des problèmes conséquents de stockage, de traitement bibliographique, de conservation, de numérisation, d'autant qu'à ce stade le numérique n'a pas freiné l'inflation des productions papier. Si globalement ces hypothèques pèsent encore peu au regard des enjeux culturels de cette collecte, il viendra peut-être un temps où le périmètre du dépôt légal devra faire l'objet d'une nouvelle réflexion, s'affranchir plus clairement de l'exhaustivité, toujours espérée, jamais atteinte.

\section{Les collections spécialisées : opportunités et défis}

La présence des collections spécialisées à la $\mathrm{BnF}$ constitue une magnifique opportunité. À la lecture de ce qui précède, on peut penser qu'elles n'ont en partage que de ne pas relever, majoritairement, de la culture du livre. À la réflexion, on peut voir qu'elles se répartissent en grands types qui font apparaître des thématiques ou des caractéristiques communes : l'importance de l'image par exemple (estampes, photographies, monnaies, médailles, entre autres), une consanguinité plus évidente avec l'archive, une complexité plus grande de signalement, le besoin d'interactions plus nombreuses en service public, des complexités de communication notamment pour les objets précieux ou en trois dimensions, des besoins particuliers en équipements et en traitements de conservation...

La présence des collections spécialisées ancre l'établissement dans une conception patrimoniale profonde, large, elle permet une historiographie passionnante, et elle a su s'adapter assez facilement à la patrimonialisation généralisée qu'ont connu les objets culturels. Elle permet aux usagers une approche qu'on peut dire d'emblée multimédia et pluridisciplinaire, qui est à leurs yeux une des principales qualités des collections de la $\mathrm{BnF}$, une des principales raisons aussi de fréquenter l'établissement.

Cette diversité se reflète bien dans la bibliothèque numérique de la $\mathrm{BnF}$, Gallica, dont le caractère multimédia (textes, images, sons, objets, image animée) est incontestablement un des points forts. Elle a permis la mise en place d'une politique de recherche ambitieuse, le plus souvent en partenariat avec des univer- sités, dont beaucoup des sujets concernent l'image fixe, la numismatique, les manuscrits, le spectacle. Cette diversité permet aussi une diffusion culturelle très riche, qui est rarement en manque de ressources internes. Elle favorise la réalisation d'expositions de toutes échelles, le développement d'une politique d'édition d'ouvrages de valorisation significative ${ }^{7}$. Institutionnellement, la $\mathrm{BnF}$ est une bibliothèque que ses partenariats et son activité quotidienne mettent en contact avec autant de musées que de bibliothèques, du fait de l'existence de ce patrimoine dans ses réserves. Elle prête environ 2000 pièces par an à des expositions françaises ou étrangères, la plupart de celles-ci relevant des collections spécialisées. Pour une bonne part, le rayonnement international de la $\mathrm{BnF}$ est ainsi issu de la qualité de ses collections hors imprimés. Un bon exemple récent est le Louvre Abu Dhabi, dont la BnF a pu rejoindre la douzaine de prêteurs français prestigieux, à l'égal du Louvre ou du Musée d'Orsay, ou encore du Centre Pompidou ou du Musée Guimet.

Enfin, ou avant tout, cette diversité des collections est aussi une richesse humaine : la présence d'autant d'éléments ne relevant pas de la culture du livre nécessite des compétences scientifiques particulières, que la BnF s'attache à chercher et à valoriser : archivistes paléographes, historiens de la gravure ou de la photographie, du cinéma ou du théâtre, numismates ou archéologues, géographes, musicologues ou historiens de la musique - alors que, on le sait, cette diversité de spécialisation a de moins en moins cours dans les cursus de formation des conservateurs ou des bibliothécaires. Il en va de même du côté de la conservation, où l'expertise de la $\mathrm{BnF}$ et de ses métiers d'art est reconnue nationalement et internationalement : ainsi pour la restauration de la photographie, ou des cartes et des globes ${ }^{8}$.

\section{De la bibliothèque à la bibliothèque-musée}

Ces opportunités ont des revers, ou représentent des défis. Les principaux concernent d'une part la « culture d'entreprise ", et d'autre part l'enjeu d'image.

En interne, le poids énorme de l'imprimé livres, périodiques -, ou plus largement de tous les 
documents textes - dont les manuscrits - nécessite une grande vigilance dans les équilibres entre les «médias » : ainsi pour tous les budgets - acquisition, conservation, numérisation -, dans la répartition des moyens humains, ou encore pour les développements informatiques relatifs aux collections. Il en va de même dans la programmation des expositions ou des manifestations.

Du côté des publics, l'identité de bibliothèque de la BnF est incontestablement un obstacle à une juste appréhension de la richesse des collections spécialisées qui pour certaines mettent la bibliothèque à la hauteur des plus grands musées français ou étrangers. Malgré des efforts stratégiques qui sont parfois couronnés d'un plein succès, à la faveur d'une entrée prestigieuse ou d'une exposition réussie, mais qui malheureusement ne parviennent guère à s'ancrer dans les esprits du fait d'une actualité culturelle foisonnante, le grand public n'attend pas toujours la BnF sur des domaines tels que la gravure, la photographie, l'archéologie, l'audiovisuel, le spectacle, la musique, la chanson ou le jeu ${ }^{9}$.

Prenant acte de cette difficulté, et après une réflexion qui n'a pas été sans débats, la direction de la $\mathrm{BnF}$ a fait le choix en 2016 de jouer pleinement la carte de la communication. Si à presque toutes les époques, la Bibliothèque nationale a été un musée qui n'en portait pas le nom, et qui de ce fait n'a pas atteint la notoriété qu'il mérite, il a paru temps de déclarer explicitement cette fonction ou cette ambition de l'institution, en donnant enfin à Richelieu, le site des départements spécialisés, le nom de «Bibliothèques Musée Galeries " ${ }^{10}$. La mutation n'est pas seulement « cosmétique ». Elle veut signer le développement d'une politique radicalement plus ouverte vers le grand public, les enseignants et les scolaires, le tourisme ${ }^{11}$. Elle sera peut-être aussi à terme le ferment d'une redistribution des identités des sites de la BnF. Si jusqu'à présent, la diffusion culturelle a joué l'entrecroisement des thèmes et des collections - par exemple le livre rare à Richelieu, la photographie ou la gravure sur le site François Mitterrand -, il n'est pas exclu de travailler un jour à élaborer des images de $\operatorname{site}^{12}$. Mais ceci anticipe largement une histoire qui va connaître à l'horizon 2021, date de la réouverture complète de Richelieu, le début d'un de ses plus intéressants chapitres.

\section{ORIENTATIONS BIBLIOGRAPHIQUES}

\section{Généralités}

- Balayé, Simone, La Bibliothèque nationale des origines à 1800, Droz, 1988

- Blasselle, Bruno et Melet-Sanson, Jacqueline, La Bibliothèque nationale de France, mémoire de l'avenir, Gallimard, 1990 (collection Découvertes)

\section{Sur les collections spécialisées}

- Bléchet, Françoise, « Le destin des globes dans les collections de la Bibliothèque royale au XVIII ${ }^{\mathrm{e}}$ siècle ", in Les Globes de Louis XIV, étude artistique, historique et matérielle, sous la direction de Catherine Hoffmann et Hélène Richard, BnF, 2012

- Catalogue des instruments scientifiques de la Bibliothèque nationale de France (à paraître cet automne), sous la direction d'Antony Turner, BnF-Brepols

- Chevrel, Claudine, "La Bibliothèque de l'Arsenal », in Revue de la société des Amis de la Bibliothèque Forney, $\mathrm{n}^{\circ}$ 185, 2010 (disponible sur Internet)

- Décollogne, Roger, La Phonothèque nationale, vingt ans d'évolution, 1940 - 1960 [Supplément : 1960 - 1963 : l'essor], Phonothèque nationale, 1960 - 1964

- Dévigne, Roger, Une «Bibliothèque nationale » sonore pour la conservation des imprimés phonographiques : la Phonothèque nationale : bilan de dix ans de travail, le dépôt légal, la lecture sonore, l'histoire et la géographie sonores, les anthologies phonographiques, Phonothèque nationale, 1949

- Giteau, Cécile, « Une centrale documentaire au service des praticiens du théâtre et de la recherche : d'Auguste Rondel à aujourd'hui ", in Le théâtre au plus près. Pour André Veinstein, Saint-Denis, Presses universitaires de Vincennes, 2005, p. 239-262

- Lesure, François, The Music department of the Bibliothèque nationale, in Notes, 1978, vol. 35, p. 251-268

- Manuscrits, xylographes, estampages : les collections orientales du département des Manuscrits, sous la direction d'Annie Berthier, $\mathrm{BnF}, 2000$

- Massip, Catherine, « La musique à la Bibliothèque nationale de France. 1 : les sources écrites », in Fontes artis musicae, 2000, vol. 47, no $2-3$, p. 104-130

- Sarmant, Thierry, Le Cabinet des médailles de la Bibliothèque nationale, 1661-1848, École des chartes, 1994

- Trésors photographiques de la Société de géographie, sous la direction d'Olivier Loiseaux, BnF-Glénat, 2007 


\section{NOTES}

1- La BnF appelle ainsi ses collections de documents autres que les livres et périodiques imprimés.

2- 1368 est la date à laquelle Charles $\mathrm{V}$ a installé sa bibliothèque dans le palais du Louvre.

3- Dépôts, dons manuels, donations, legs, acquisitions, dépôt légal, saisies notamment révolutionnaires, échanges, ce dernier mode n'ayant plus cours.

4- On connaît sans doute la belle formule : « ... nous avons délibéré de faire retirer, mettre et assembler en notre librairie toutes les œuvres dignes d'être vues qui ont été ou qui seront faites... de notre tems pour avoir recours auxdits livres, si de fortune ils étoient cy après perdus de la mémoire des hommes... ».

5- Il s'agit globalement de l'ensemble des sites dont le serveur est installé dans le périmètre des frontières nationales. Une seule collecte par robot de ces sites représente environ 50 téraoctets de données. On laisse ici de côté la plus grande partie du champ numérique - par exemple les logiciels ou le jeu multimédia, qui mériteraient à eux seuls de larges développements.

6- Dans les multimédias comptent les multimédias multisupports, un très vaste ensemble de documents composites, souvent pédagogiques et où figurent beaucoup de productions pittoresques, par exemple tous les coffrets d'apprentissage de type «boîte du petit chimiste », ou encore l'initiation à l'œnologie avec, cela va de soi, les échantillons de dégustation...

7- Voir, pour ne prendre qu'un exemple, le succès de l'ouvrage Animal sur la représentation des animaux, paru en 2014 et préfacé par Michel Pastoureau, très bien accueilli par la presse et par le public.

8- Les ateliers de restauration de la BnF se voient parfois confier des collections de bibliothèques françaises ; ils interviennent aussi à l'étranger, quand les objets considérés requièrent des compétences introuvables sur place.

9- Une récente étude de notoriété, réalisée avec la société Ifop, atteste que la $\mathrm{BnF}$ est d'abord perçue comme un lieu de recherche et de savoir, avant d'être un « lieu culturel ». Il est patent que les principales marges de progrès en termes de fréquentation concernent l'évolution de cette image : faire toujours mieux connaître la BnF comme lieu d'expositions, de colloques et de débats, d'événements.

10- Le pluriel de bibliothèques se justifie par la présence sur le site de la bibliothèque de l'Institut national d'histoire de l'art installée salle Labrouste, et de la bibliothèque de l'École nationale des chartes. Le terme de Galeries, quant à lui, fait allusion aux galeries d'expositions, mais aussi à une volonté forte du projet de s'inscrire dans un paysage urbain de passages et de galeries, qui s'étend du Louvre à l'Opéra de Paris.

11- Ces quelques lignes n'épuisent pas un sujet très vaste et passionnant. $\mathrm{La} \mathrm{BnF}$ envisage de monter, dans les années qui viennent, un séminaire, puis un colloque international, sur la question de la relation entre bibliothèque et musée, ainsi que sur celle des bibliothèques-musées.

12- Une hypothèse évoquée serait de pousser plus fortement François Mitterrand comme lieu des savoirs et Richelieu comme lieu de la création.

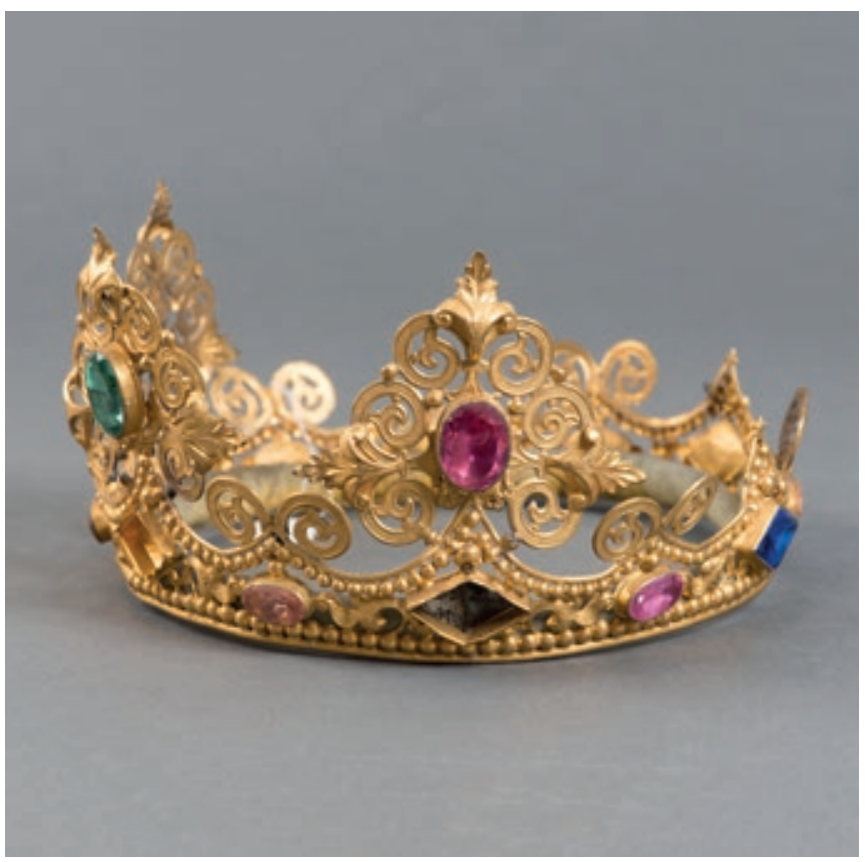

Un des très nombreux bijoux de scène conservés à la Bibliothèque-musée de l'Opéra, ici une couronne pour l'opéra Tannhaüser, création d'Alfred Albert, costumier (1861) 